\title{
Research \\ Chromosomal abnormalities in patients with recurrent spontaneous pregnancy loss and sub-fertility
}

\author{
Vajira H W Dissanayake ${ }^{1,2}$, A M Maheshi H Athapaththu ${ }^{1}$, D Jeewani S Opanayake ${ }^{1}$, Nethra T Hidellage ${ }^{1}$, \\ Kalpani Wijetunge ${ }^{1}$, Christeen R J Pedurupillay ${ }^{1}$
}

Sri Lanka Journal of Obstetrics and Gynaecology 2009; 31: 84-87

\begin{abstract}
Introduction: Chromosomal abnormalities are implicated in the etiology of recurrent spontaneous pregnancy loss and sub-fertility.

Objective: To describe the chromosomal abnormalities detected in a series of patients with recurrent spontaneous pregnancy loss and sub-fertility tested in our laboratory.

Methods: 442 patients including 348 patients (171couples, 6 women) with recurrent spontaneous pregnancy loss (Group A), 58 patients (24 couples, 4 men, 6 women) with sub-fertility (Group B), 36 patients (18 couples) with IUI/ IVF failure (Group C) were karyotyped.

Results: Chromosomal abnormalities were detected in $42(9.5 \%) .21(50 \%)$ were male and $21(50 \%)$ were female. These abnormalities include $33(79 \%)$ structural abnormalities, 9 $(21 \%)$ numerical abnormalities. All those who had numerical abnormalities were mosaics for normal and abnormal cell lines. The rate of abnormalities seen in groups A, B and C were 8.3\% [29/348], $10.4 \%$ [6/58], and $19.4 \%$ [7/36] respectively. There was a significant difference between the chromosomal abnormalities seen in Group $C$ when compared with combined groups $\mathrm{A}$ and $\mathrm{B}(\chi 2=4.5, \mathrm{df}=1, \mathrm{p}=0.34)$.

Conclusions: The detection of a chromosomal abnormality in one partner of a couple experiencing recurrent spontaneous pregnancy loss or sub-fertility alters the management of the couple. Therefore such couples should be karyotyped before they are offered assisted reproductive interventions.
\end{abstract}

\footnotetext{
${ }^{1}$ Asiri Centre for Genomic and Regenerative Medicine, Asiri Surgical Hospital, Colombo.

${ }^{2}$ Human Genetics Unit, Faculty of Medicine, University of Colombo.

Corresponding author: Vajira H W Dissanayake

E-mail:vajirahwd@hotmail.com
}

Key words: Chromosomal abnormalities, recurrent spontaneous pregnancy loss, sub-fertility.

\section{Introduction}

Although country wide estimates are not available, anecdotal evidence suggest that recurrent spontaneous pregnancy loss and sub-fertility affect a significant proportion of couples in Sri Lanka. While the aetiology of these two conditions is varied ${ }^{1}$, biologically these are two pregnancy related outcomes by which nature prevents the birth of a child with a birth defect to a couple where one or both partners harbor a chromosomal abnormality. There is however, no consensus on the number of spontaneous pregnancy losses a couple must experience before undergoing genetic evaluation or when a couple with sub-fertility should undergo genetic evaluation. As such genetic testing tends to take a back seat especially in countries like Sri Lanka where such testing is not freely available.

\section{Objective}

In this paper we describe the chromosome abnormalities seen in couples with recurrent spontaneous pregnancy loss and sub-fertility tested in our laboratory.

\section{Materials and methods}

We prospectively maintained phenotyping and karyotyping data of all patients tested in our laboratory from January 2006 to March 2009. These patients were divided into three groups for the purpose of this analysis: couples with recurrent spontaneous pregnancy loss (two or more spontaneous pregnancy losses) who have not had assisted reproductive interventions such as intrauterine insemination or in vitro fertilization (Group A); couples with sub-fertility who have not had assisted reproductive interventions (Group B); and couples with recurrent spontaneous pregnancy loss or sub-fertility who have been treated with assisted reproductive interventions without success (Group C). 
Karyotyping was performed in these patients using standard methods. Briefly it involved obtaining a sample of peripheral blood by venepuncture; culturing the lymphocytes in peripheral blood using a culture medium; arresting mitosis at metaphase by adding a spindle blocker; harvesting chromosomes; slide preparation; staining of slides with Geimsa or Leishman stain; and karyotyping using an automated karyotyping system. Standard harvesting procedures were performed on samples tested up to March 2008. Thereafter all samples were subjected to high resolution karyotyping. At least 20 chromosome spreads were counted and analysed for each patient. There was no significant difference in the number of chromosomal abnormalities detected using the two methods, therefore the combined data is presented in the results table.

\section{Results}

A total of 442 patients were tested. There were 348 in group A (171 couples; 6 females); 58 in group B ( 24 couples; 4 males; 6 females); and 36 in group C (18 couples). Group A and C included those with two or more recurrent spontaneous pregnancy losses.

Chromosomal abnormalities were detected in 42 $(9.5 \%) .21(50 \%)$ of them were male and $21(50 \%)$ were female. $33(79 \%)$ had structural chromosomal abnormalities. 9 (21\%) had numerical chromosomal abnormalities. The abnormalities detected are summarized in table 1. All those who had numerical abnormalities were mosaics for normal and abnormal cell lines. The abnormalities marked with an asterisk $\left({ }^{*}\right)$ are considered to be polymorphic chromosomal variants rather than true abnormalities but are listed here because they tend to be seem more often in adverse pregnancy related outcomes such as recurrent spontaneous pregnancy loss and sub-fertility.

Table 1. Summary of karyotypic abnormalities

\begin{tabular}{lcr}
\hline Karyotype & Indication & No \\
\hline $45, \mathrm{X} / 46, \mathrm{X}$ & $\mathrm{RPL}$ & 1 \\
$45, \mathrm{X} / 47, \mathrm{XXX}$ & $\mathrm{RPL}$ & 1 \\
$45, \mathrm{X} / 45, \mathrm{i}(\mathrm{X}) / 46, \mathrm{XY}$ & $\mathrm{RPL}$ & 1 \\
$46, \mathrm{XX} / 46 \mathrm{X}, \mathrm{r}(\mathrm{X})$ & $\mathrm{RPL}$ & 1 \\
$46, \mathrm{XX} / 47, \mathrm{XX}+17$ & $\mathrm{RPL}$ & 1 \\
$46, \mathrm{XY} / 47, \mathrm{XX},+$ marker & RPL & 1 \\
$46, \mathrm{XX}, \operatorname{dup}(4)(12 \mathrm{q}-\mathrm{q} 21)$ & $\mathrm{RPL}$ & 1 \\
$46, \mathrm{XY}, \operatorname{del}(12)(\mathrm{q} 13-\mathrm{q} 14)$ & $\mathrm{RPL}$ & 1 \\
$46, \mathrm{XX}, \mathrm{t}(7 ; 14)(\mathrm{q} 36 ; \mathrm{q} 11.2)$ & $\mathrm{RPL}$ & 1 \\
$46, \mathrm{XY}, \operatorname{inv}(9)(\mathrm{p} 11 ; \mathrm{q} 13)$ & $\mathrm{RPL}$ & 1 \\
& & (Continued)
\end{tabular}

\begin{tabular}{|c|c|c|}
\hline Karyotype & Indication & No \\
\hline $46, \mathrm{XX}, 9 \mathrm{qh}+*$ & RPL & 2 \\
\hline $46, \mathrm{XX}, 13 \mathrm{ps}+, 14 \mathrm{ps}^{*}$ & RPL & 1 \\
\hline $46, \mathrm{XX}, 14 \mathrm{ps}^{+*}$ & RPL & 1 \\
\hline $46, \mathrm{XY}, 14 \mathrm{ps}+^{*}$ & RPL & 1 \\
\hline $46, X X, 15 p+*$ & RPL & 1 \\
\hline $46, \mathrm{XX}, 21 \mathrm{ps}+^{*}$ & RPL & 3 \\
\hline $46, \mathrm{XX}, 22 \mathrm{ps}+^{*}$ & RPL & 2 \\
\hline $46, \mathrm{XY}, 14 \mathrm{ps}+, 21 \mathrm{ps}+^{*}$ & RPL & 1 \\
\hline $46, \mathrm{XY}, 22 \mathrm{ps}+^{*}$ & RPL & 4 \\
\hline $46, X X, 22$ pstk+* & RPL & 1 \\
\hline $46, X Y, Y q h+*$ & RPL & 2 \\
\hline $47, X X X / 45, X$ & SF & 1 \\
\hline $46, \mathrm{XX}, 9 \mathrm{qh}+*$ & SF & 1 \\
\hline $46, X Y, 21 p s+*$ & SF & 1 \\
\hline $46, \mathrm{XY}, \mathrm{Yqh}+{ }^{*}$ & SF & 3 \\
\hline $46 \mathrm{XX} / 45, \mathrm{X}$ & RPL \& IVF $\times 1$ & 1 \\
\hline $46, X Y, 13 p s+*$ & RPL \& IVF × 3 & 1 \\
\hline $45, X / 46, X X$ & SF - IUI × 2 & 1 \\
\hline $46, X Y / 47, X X Y$ & SF $-I V F \times 2$ & 1 \\
\hline \multicolumn{3}{|c|}{$46, X Y, \operatorname{del}(1)(q 42-q 44)[2] /$} \\
\hline $46, X Y[11]$ & $\mathrm{SF}-\mathrm{IUI} \times 10 ; \mathrm{IVF} \times 3$ & 1 \\
\hline $46, \mathrm{X}, \mathrm{t}(\mathrm{Y} ; 15)$ & SF - IUI $\times 2$ & 1 \\
\hline $46, \mathrm{XY}, \mathrm{Yqh}+*$ & SF - IUI × 1 & 1 \\
\hline
\end{tabular}

RPL - Recurrent spontaneous pregnancy loss; SF - Subfertility; IUI - Intrauterine Insemination; IVF - In vitro Fertilisation; *Polymorphic chromosomal variants

The rate of abnormalities seen in groups A, B, and $C$ were $8.3 \%$ [29/348], $10.4 \%$ [6/58], and $19.4 \%$ [7/36] respectively. There was a significant difference between the chromosomal abnormalities seen in Group $\mathrm{C}$ when compared with combined groups $\mathrm{A}$ and $B\left(\chi^{2}=4.5, d f=1, p=0.34\right)$.

\section{Discussion}

The objective of this paper was to describe the chromosomal abnormalities found in couples with recurrent spontaneous pregnancy loss and subfertility. Overall $9.5 \%$ of males and females tested had a chromosomal abnormality with equal numbers of males and females having abnormalities. Although historically recurrent spontaneous pregnancy loss is defined as three or more pregnancy losses, current data 
suggest that there is no difference in the frequency of chromosomal abnormalities between couples with two compared to those with three or more pregnancy losses $^{2}$. The rate of chromosomal abnormalities observed in couples with recurrent spontaneous pregnancy loss observed by us was higher than that reported in a larger series in India ${ }^{3}$. This analysis was expanded to examine whether couple who have had failed assisted reproductive interventions were more likely to have chromosomal abnormalities than those who have not had such interventions which showed that in fact chromosomal abnormalities were more common in such couples. Therefore chromosome culture and karyotyping should be considered in the investigation of couples with recurrent spontaneous pregnancy loss or sub-fertility at least at the time of offering assisted reproductive interventions if not earlier. This observation is in keeping with observations from other studies ${ }^{4}$.

Interestingly there were a significant number of patients with chromosomal abnormalities which are considered to be normal polymorphic chromosomal variations rather than true chromosomal abnormalities per say. Such normal polymorphic variants however, are believed to contribute to the instability of chromosomes during meiosis with a tendency towards an increased risk of aneuploidy resulting in recurrent spontaneous pregnancy loss or sub-fertility ${ }^{5}$. In fact in our laboratory such variants are observed almost exclusively only in these two groups. Figure 1 illustrates a family in which such an abnormality was observed in a 27 year old woman who experienced four recurrent spontaneous pregnancy losses. She had a younger brother. Her mother gave a history of not being able to conceive further in spite of wanting to have more children. In fact the age gap between the two children was 19 years. The mother had three younger sisters who were all sub fertile. In a clinical setting investigation of such extended families is not possible to verify whether the chromosomal abnormality observed in the 27 year old woman was familial. This family however, illustrates the usefulness of the family history as a tool to identify families who may be at increased risk of carrying chromosomal abnormalities. If this family history was picked up soon after the first pregnancy ended in an abortion and appropriate assisted reproductive interventions instituted then the mental, physical and financial anxieties that the couple had to undergo over a period of several years could have been avoided ${ }^{6,7}$. The family history, although often underutilized, is a useful tool that can be effectively used to pick up couples with recurrent spontaneous pregnancy loss and sub-fertility who would benefit from chromosomal culture and karyotyping.
Figure 1. Family History

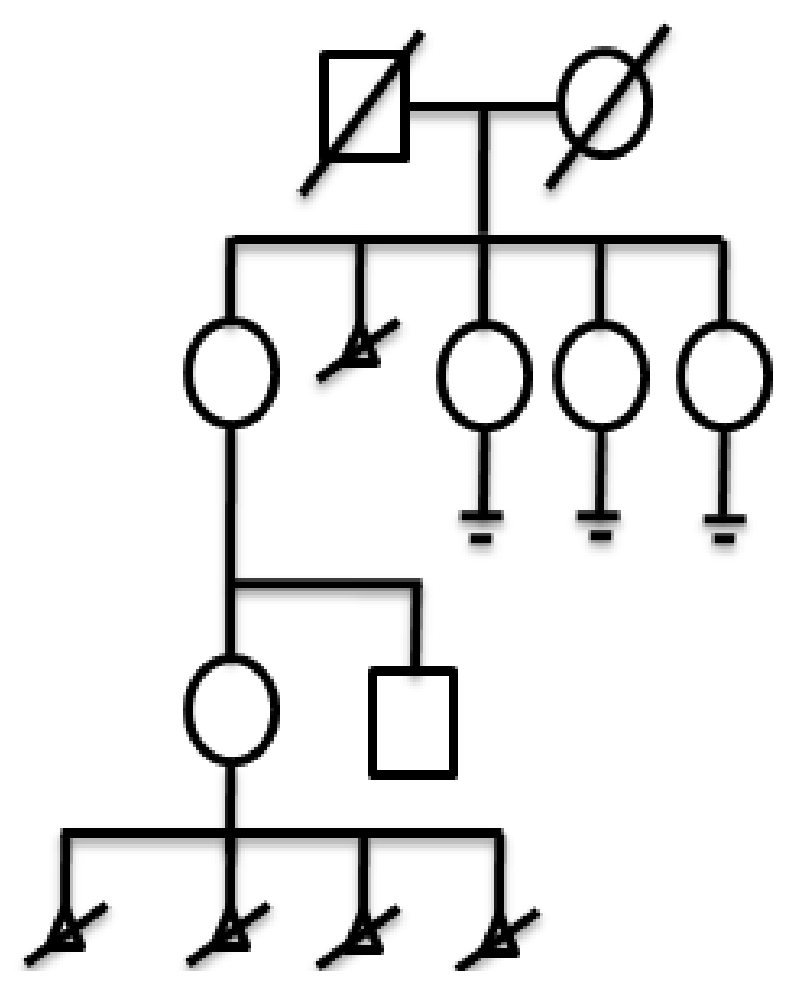

Karyogram of the Proband showing 9qh+

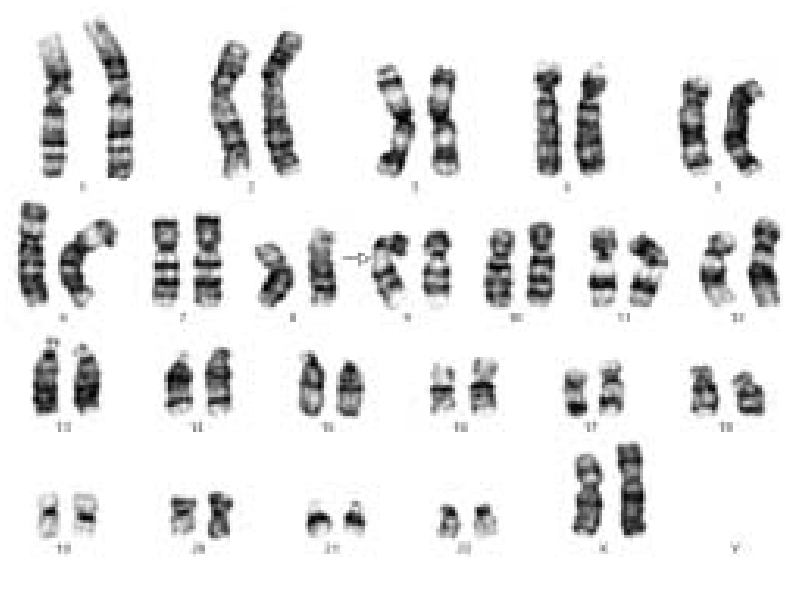

$46, X X, 9 q h+$

\section{References}

1. Habayeb OM, Konje JC. The one-stop recurrent miscarriage clinic: an evaluation of its effectiveness and outcome. Hum Reprod 2004; 19: 2952-8.

Sri Lanka Journal of Obstetrics and Gynaecology 
2. Jaslow CR, Carney JL, Kutteh WH. Diagnostic factors identified in 1020 women with two versus three or more recurrent pregnancy losses. Fertil Steril 2009; [Epub ahead of print].

3. Dubey D, Chowdhury MR, Prahlad B, Kumar V, Mathur R, Hamilton S, Kabra M, Menon PSN, and Verma IC. Cytogenetic causes for recurrent spontaneous abortions an experience of 742 couples (1484 cases). Indian J of Hum Genet 2005; 11: 94-8.

4. Stern C, Pertile M, Norris H, Hale L, Baker HW.
Chromosome translocations in couples with in-vitro fertilization implantation failure. Hum Reprod 1999; 14: 2097-101.

5. Ward KJ. Genetic factors in recurrent pregnancy loss. Semin Reprod Med 2000; 18: 425-32.

6. Rai R, Regan L. Recurrent miscarriage. Lancet 2006; 368: 601-11.

7. Kaluarachchi A. Infertility management services in Sri Lanka - Are we doing enough? Sri Lanka J of Obstet and Gynecol 2008; 30: 1-2. 